\title{
Competencias en educación: Ideas para el diseño curricular desde la deliberación práctica y crítica, basada en el desarrollo humano y la transformación social
}

\section{Competences in Education: Curricular Design Ideas from Critical and Pctical-Deliberation based on Human Development and Social Transformation}

\author{
María Isabel Araya Muñoz. \\ Escuela de Secretariado Profesional \\ Universidad Nacional de Costa Rica \\ Heredia, Costa Rica \\ isaaraya@yahoo.com
}

Recibido 01 de noviembre de 2010 • Aceptado 09 de marzo de 2011

\begin{abstract}
Resumen. Los sistemas educativos, en América Latina y en el mundo entero, están siendo llamados a dar respuesta a las exigencias de profesionales altamente formados, que respondan a los conocimientos y habilidades emergentes exigidas por los avances científicos y tecnológicos. Ante esas peticiones de la realidad económica y productiva, las competencias se han convertido en el estandarte para que los sistemas educativos planteen sus propuestas curriculares. En estas se manifiesta la ampliación de los contenidos al aprender funciones o tareas con base en adaptaciones de otros contextos, desde la experticia y sin estudios profundos de la realidad de las fuentes curriculares. En este artículo se plantea la importancia de considerar que un proyecto educativo basado en competencias profesionales se debe visualizar como un proceso de formación de la persona, de manera integral. En el análisis de esa proposición se discute el abordaje de las competencias desde la educación, el cual ha pretendido relacionar la teoría y la práctica, acercando los contenidos académicos al hacer en un puesto de trabajo, en divergencia con la necesidad de razonar las competencias en educación como el medio para integrar el conocimiento y la experiencia. No se trata solo de resolver tareas específicas en un futuro puesto de trabajo, sino también de la búsqueda de soluciones de los problemas en los diferentes ámbitos de la vida. Para el logro de esa visión integradora de la educación, se proponen algunas ideas para diseñar proyectos curriculares por competencias desde la tradición práctica deliberativa y crítica, con una concepción curricular humanista y de transformación social.
\end{abstract}

Palabras claves. Diseño curricular, competencias, innovación educativa, aprendizaje, educación, diseño curricular práctico-deliberativo y crítico, desarrollo humano, transformación social.

Licenciada en Educación Comercial. Máster en Educación y egresada de Magíster en Planificación Curricular, Universidad de Costa Rica. Académica: Escuela de Secretariado Profesional. Universidad Nacional de Costa Rica. Heredia, Costa Rica. 
Abstract. Educational systems in Latin America and worldwide are being required to provide highly qualified professionals, with the knowledge and abilities needed according to the scientific and technological advances. In view of such demands of the economic and productive reality, the educational systems present their curricular proposals under the flag of competencies. Such proposals have increased the contents, since functions or tasks are adapted from other contexts, based on expertise, without deeper studies on the real situation of the curricular sources. This paper demonstrates the importance of considering that an educational project based on professional competencies should be visualized as the individual's comprehensive educational process. The competences approach is discussed from the point of view of education, which includes theory and practice, bringing closer the academic contents and the job activity, differing from the necessity of reasoning the competencies in education as a means to combine knowledge and experience. It is not only about being able to manage specific tasks in a future job, but about also finding solutions to problems that may arise in the different spheres of life. Aiming at such comprehensive vision of education, some ideas are proposed to design curricular projects based on competencies, from the practical-deliberative and critical tradition, focused on a humanist curricular concept and social transformation.

Keywords. Curricular design, competencies, educational innovation, learning, education, curricular practical-deliberative and critical design, human development, social transformation.

\section{Introducción}

La aplicación de las competencias desde los ámbitos del trabajo y de la escuela se ha llevado a cabo, en el primer caso, con miras a la mejora de la producción en una empresa, y en el segundo, a formar personas calificadas para un futuro puesto de trabajo.

Esa situación no es casual. En los últimos años, los países desarrollados han incursionado en un cambio hacia lo global y la transnacionalización de la economía, por lo que se demanda una transformación en los requerimientos mundiales de productividad y de calidad. Aunado a esto, se deben considerar los avances científicos y tecnológicos que se incorporan a los procesos productivos y que exigen también nuevas capacidades y habilidades humanas. En este contexto, Tünnermann (2008) afirma que el mundo productivo les plantea inquietudes a los sistemas educativos y al desarrollo sostenible de la región de América Latina. Por esta razón, el perfil profesional de los estudiantes y las estudiantes requiere de una modificación a partir de las exigencias de competitividad de las empresas y de las nuevas representaciones socioculturales.

En ese sentido, las universidades se encuentran frente a un reto: responder con planes de estudio relevantes y pertinentes, de acuerdo con los avances científicos y las tecnologías de la comunicación, que han generado lo que Victoria González define como “(...) tres revoluciones: -sociocultural, tecnológica y económica-(...)" (González, 2005, p. 3). Estas vienen a modificar las formas de deliberar y de aportar ideas para la resolución de problemas, la forma o habilidad para resolverlos y los procedimientos institucionales.

La idea de esta reflexión es considerar que un proyecto educativo basado en competencias profesionales se debe visualizar como un proceso de enseñanza y de aprendizaje. No se trata solo de que la persona aprenda para la tarea, sino que participe en una formación para el desempeño eficiente y eficaz en el ámbito profesional; y esté formado holísticamente para el disfrute de la vida personal, familiar, laboral y comunal. Esa perspectiva del currículo se logra a través del diseño de proyectos con base en una deliberación práctica y crítica de la realidad, donde se conciba el currículo desde el desarrollo humano y desde la transformación social. 
En el análisis de esa proposición se discute el abordaje de las competencias desde la educación. Este se ha realizado con miras a relacionar la teoría y la práctica, acercando los contenidos académicos al puesto de trabajo, en divergencia con la necesidad de considerar las competencias en educación como el medio para integrar los conocimientos teóricos y prácticos para potenciar, en la persona, el desarrollo de sus capacidades, habilidades y valores para construir un proyecto de vida con éxito, y participar en la familia y en la comunidad con liderazgo.

En segundo lugar y para el logro de esa visión integradora de la educación, se proponen algunas ideas para diseñar proyectos curriculares por competencias, desde la tradición práctica deliberativa y crítica, con una concepción curricular humanista y de transformación social.

\section{Las competencias en educación}

Los estudios sobre competencias son múltiples en entornos nacionales e internacionales. Su interés es el análisis del desempeño en torno a la eficiencia de las tareas en un puesto de trabajo y, en el ámbito educativo, con la intencionalidad de corresponder la actividad de aula con la realidad laboral. Esas relaciones remiten a dos áreas fundamentales: el trabajo y la educación. Lo anterior refiere a dos intereses, el laboral y el profesional, que aislados se orientan hacia fines diferentes, uno el económico o a la producción y el otro, al desarrollo integral de la persona.

Con base en algunos trabajos realizados en las últimas tres décadas, a continuación se presenta, de manera muy somera, la idea básica de la conceptualización de las competencias.

Mcclelland (1973),

citado por Levy-Levoyer (1997)

Riesco (2008)
Competencia personal de quienes ocupan determinados puestos de trabajo, considera los factores de cualificación, habilidades, destrezas, capacidades y talante ante sí mismo.

La Organización para la Cooperación y Desarrollo Económico (OCDE), en el documento DESECO (Definition and Selection of competentes (OCDE, 2002:4), define la competencia como "La habilidad para responder a las demandas o llevar a cabo tareas con éxito y consistentes con las dimensiones cognitivas y no cognitivas". (p. 85)

Villarini (1996)

La persona competente tiene la información y conceptos, sabe lo que hace, por qué lo que hace y conoce el objeto sobre el que actúa; capacidad de ejecución (integración del conocimiento procesal o las destrezas intelectuales y psicomotoras para ejecutar). Resume el ser competente: tener actitud o disposición, conocimiento declarativo y procesal y actuar de la manera que se considera correcta.

Levy-Levoyer (1997)

Refiere las competencias a tareas o situaciones de trabajo y a la regulación de la que es capaz el operario en el entorno de su trabajo y de su actividad. Están vinculadas a una tarea o una actividad determinada que también pueden aplicarse a un conjunto de actividades.

Mertens (2000)

Es capacidad demostrada por una persona para lograr un resultado, en concordancia con los objetivos y el contexto global de la organización. Orientación aprendizaje-desempeño.

Continúa... 
Vargas (s. f., p. 17)

Catalano, Avolio, y Sladogna (2004)

Villa y Poblete (2004)

Proyecto Tuning América Latina (2005)

Tobón (2005)
Arias y Charpentier (2004)

“... designa a todo un conjunto de características de las personas que las hace exitosas en la realización de un trabajo. Por su carácter están ligadas estrechamente con el contexto laboral, por lo que las Competencias son mucho más dinámicas, fluidas y complejas que los constructos psicológicos tradicionales (...)"

Las competencias laborales pueden ser definidas como el conjunto identificable y evaluable de capacidades que permiten desempeños satisfactorios en situaciones reales de trabajo, de acuerdo con los estándares históricos y tecnológicos vigentes.

La posibilidad de aumento en el rendimiento y la competitividad de la empresa dentro del mercado que ha formado sus colaboradores mediante este sistema

En el marco del Proyecto de Competencias de la Universidad de Deusto, definen competencias como un buen desempeño en contextos complejos y auténticos, basada en la integración y activación de conocimientos, habilidades y destrezas, actitudes y valores.

Las competencias describen los resultados del aprendizaje, lo que una persona estudiante sabe o puede demostrar una vez completado un proceso de aprendizaje.

Relaciona las competencias con un saber hacer reazonado para hacer frente a la incertidumbre: manejo de incertidumbre en un mundo combiante en lo social, lo político y lo laboral, dentro de una sociedad globalizada y en continuo cambio. Se deben abordar como estructura compleja de atributos necesarios para el desempeño en situaciones diversas, donde se combinan conocimiento, actitudes, valores y habilidades, con las tareas que se tienen que desempeñar en determinadas situaciones.

Gimeno (2009)

Relaciona las competencias con pericia, posibilidad, estar dotado para algo, realizar, llevar a cabo, ser capaz de responder a requerimientos para resolver problemas.

Desde esas entidades, las competencias están asociadas con la capacidad para realizar los roles de trabajo, de acuerdo con los niveles requeridos en el empleo, y se expresan mediante el dominio de una tarea, es decir, las capacidades y habilidades terminales de un título profesional. Es un conjunto de realizaciones profesionales con valor y significado en el empleo, cuando se asume el rol de una ocupación, en cuya cualificación profesional es necesario alcanzar los objetivos educativos y un cierto nivel de conocimientos culturales, científicos y tecnológicos medibles en términos de desempeño intelectual, habilidades, destrezas y actitudes en un puesto de trabajo.

Ese abordaje de las competencias pretende eliminar la brecha entre la escuela y la empresa, por ello han sido harto estudiadas, con el fin de legitimizar la necesidad de formar para la tarea, a diferencia de los programas de estudio orientados hacia la formación de contenidos, estos se amplían a la función.

Las competencias se han convertido en el emblema de los sistemas educativos para darle utilidad al conocimiento. Gimeno (2009) indica que los planteamientos en esta modalidad de aprendizaje versan en darle funcionalidad a lo aprendido, saber hacer en un puesto de trabajo. Pero su propósito va más allá, pues se pretende que las competencias actúen como guías para la confección y desarrollo de los currículos, y de las políticas educativas. 
Por tanto, es importante reflexionar sobre los fines, metas o ideas de la educación, concretada en un proyecto educativo, cuya orientación es al aprender, es decir: ¿qué aprender?, ¿cómo aprenderlo? y ¿para qué aprenderlo? Las respuestas dependen del significado de la educación. En tal sentido, Kemmis (1998) indica que desde la aparición de la educación de masas, la planificación curricular ha tratado de transformar la sociedad mediante cambios en los currículos y en la escolarización; por ello el currículo no debe considerarse solo como la organización de lo que debe ser enseñado y aprendido.

Desde la educación, las competencias deben ser el medio para integrar los conocimientos teóricos y prácticos, potenciando a la persona para tomar decisiones acertadas, accionar con autonomía, expresarse con libertad, vivir con justicia y respeto a sí mismo y a los demás, realizar un proyecto laboral con éxito y participar en la familia y en la comunidad con liderazgo.

La educación así orientada permite innovar, es decir, aprender de la realidad para transformarla, mediante el diseño de un proyecto curricular por competencias, basado en el análisis de los problemas sociales. Este es el tema que se pretende desarrollar en el siguiente punto, con miras a proponer algunas ideas que se pueden tomar en cuenta en una reforma.

\section{El diseño curricular por competencias}

El currículo es una guía, un plan estratégico que Coll (1991) considera como un instrumento útil para orientar la práctica pedagógica, una ayuda para la persona docente y que debe tener en cuenta las condiciones reales en las que va a llevarse a cabo un proyecto formativo. Esta guía se plasma en un diseño curricular.

De esa manera y al ser la educación un proceso individual y colectivo, y el conocimiento, una construcción social, es necesario considerar la perspectiva para la elaboración del currículo, representada por la forma de interpretar la realidad y construir conocimiento, mediante un proceso investigativo en el que prevalece el enfoque dialógico de sus representantes.

En este apartado, se plantea la pertinencia de orientar los diseños curriculares basados en competencias profesionales, desde la tradición práctica deliberativa y crítica, con la concepción curricular basada en el desarrollo humano y en la transformación social. Villarini (1996) admite su complementariedad, tomando en cuenta que la formación profesional se visiona desde la integración de conocimientos, habilidades, destrezas y valores que la persona requiere para el disfrute de la vida en todos los ámbitos: persona, familia, trabajo y comunidad. Esta disposición de la formación profesional la define Alpízar (2008) como una alternativa estratégica para el desarrollo de los países que pretenden evolucionar y alcanzar un mejor desarrollo integral de sus pueblos, en las condiciones económicas y sociales imperantes en la actualidad.

Las instituciones educativas de nivel técnico y superior, que pretenden emprender modelos de desarrollo curricular por competencias -así definidos los proyectos educativos basados en competencias por Díaz (2005)-, deben diagnosticar, con una visión desde adentro, es decir, desde las fuentes del currículo, la persona, la sociedad, la naturaleza y la praxis, los problemas de la realidad específica que se quiere formar. Esto, porque los proyectos curriculares, en su mayoría, obedecen a parámetros educativos derivados de las necesidades del sector productivo, y no a propuestas deliberantes y críticas que defiendan los fines más esenciales de la educación y que, de esta manera, no acepten las prescripciones del interés económico mundial. Ante la actual realidad, todo ello podría constituirse en una novedosa renovación, que en palabras de Gimeno Sacristán se conceptúa como: "No es moderno aquello que es reciente o novedoso, o hacemos que lo parezca, sino lo que perdura 
y transforma la vida y la realidad. No se cambia el mundo para hacerlo progresar sin tener en cuenta cómo es, quiénes están en él y cómo lo viven" (Gimeno, 2009, p. 9).

La referencia se remite hacia las entidades de educación técnica y superior, pues se considera que, en la formación pública costarricense, con miras a mejorar la calidad, se gestan y se encuentran en proceso de planificación, propuestas curriculares basadas en competencias, las cuales corresponden a adaptaciones de países europeos, estadounidenses y latinoamericanos, sin mayor análisis de la realidad y elaboradas desde la experticia.

Por lo anterior, es importante considerar que las competencias en educación van más allá de una mera formación para el trabajo. Aunque no son una idea nueva, según Díaz (2005), desde hace más de cuatro décadas se promueven proyectos educativos basados en competencias, los cuales han tenido muy poca resonancia debido a que prevalecen las proposiciones curriculares con énfasis en modelos técnicos. Pese a lo anterior, la motivación de este estudio ha sido retomar las competencias con fines de promoción y del desarrollo de particularidades en una interacción de aprendizaje, desarrollo de habilidades, conocimientos y valores integrados con la cultura laboral y para la vida.

Ante las exigencias de recurso humano competitivo y capaz de atender las tareas emergentes de los sistemas productivos, tal y como lo indica Díaz (2005), en el campo del desarrollo curricular se han generado proyectos, de los cuales, entre las más relevantes se menciona el currículo por competencias, la flexibilidad curricular, los enfoques administrativos de planeación estratégica, el análisis institucional o de calidad total y excelencia, los de currículo basado en el constructivismo psicopedagógico, los de incorporación de las tecnologías de la información y comunicación, entre otros. En estos no encuentra una reflexión profunda sobre sus implicaciones, ni una previsión clara de su incorporación a las estructuras curriculares o a la realidad del aula, ni se ha reflexionado sobre la concepción de innovación.

Considera que esos modelos no han comprendido que innovar en educación conlleva un cambio profundo en paradigmas y prácticas sociales y educativas, producto de la reflexión y apropiación de las personas involucradas en el proceso; por ello, el discurso de esos modelos es una manifestación del pensamiento corporativo o empresarial, gestado desde hace más de cuatro décadas y que han llegado a la imposición e implantación de proyectos curriculares con un enfoque de experto y la anulación de la opinión de los artífices del contexto educativo.

Ante la orientación de los sistemas educativos de propuestas de desarrollo curricular elaboradas desde modelos técnicos, prescritas desde los tomadores de decisión, sin consulta del estudiantado, del equipo docente, de las personas empleadoras, ni de la comunidad, su naturaleza y cultura, es importante repensar: ¿Cuáles son los fines de la educación? ¿Cuáles son las ideas que sustentan los diseños curriculares?

La educación pública, interés de la autora de este artículo, debe responder a la concepción de persona que desea formar, su fin debe ser la calidad humana y redundar en los saberes que propone la UNESCO, según Delors et al. (1996): saber, saber hacer, saber ser y saber convivir. De ahí la importancia de la deliberación profunda de los problemas cotidianos desde la óptica de los involucrados y las involucradas en el proceso educativo y de las necesidades de sus contextos y fuentes del currículo.

Por su parte Pérez (2009), considera que pensar en proyectos curriculares por competencias comprende reinventar la escuela; concebir la concepción, diseño y concreción del currículo; cambiar el desarrollo del currículo en cuanto a enseñar y aprender; crear nuevos ambientes de aprendizaje y nuevas formas de evaluar, por ende, la función del docente debe ser renovada.

Esa orientación es la que se espera en los proyectos de formación de profesionales competentes: apuntar a la prosperidad del ser humano. Se debe evitar lo que Morales (2009) comenta sobre la 
realidad mexicana en el abordaje por competencias, donde, según él, se favorece el patrón de profesional enajenado, egoísta y centrado en su pequeño mundo.

"La luz es más luz sólo en la oscuridad" es la frase que utiliza Morales (2009, p. 201) al puntualizar que esa es la lógica de la tendencia actual de formación por competencias con planes diseñados con fines de construir puentes entre la escuela y el trabajo, por medio de currículos tradicionales academicistas. Plantea, también, que al llevar las competencias a la educación demanda el reconocimiento de las peticiones y necesidades de la sociedad, en su concepto más incluyente: de las minorías y la de grupos con necesidades especiales. Así, la educación se proyecta con carácter propositivo, innovador y transformador de la realidad.

Retomando la pregunta: ¿Cuáles son los fines de la educación?, que resuena en las mentes de los educadores y las educadoras cuando reciben programas de estudio elaborados desde las autoridades, con indagación de la realidad de forma muy limitada, con adaptaciones sin mayor análisis. Por el contrario, el énfasis debe ser la necesidad de que mediante los proyectos educativos se pretenda dar respuestas a los problemas humanos y establecer un vínculo entre la educación y los factores políticos, económicos y sociales. Por tanto, es necesario repensar la educación y su papel en el desarrollo de la sociedad.

La educación es una actividad propositiva, innovadora y transformadora. Esta no es una idea nueva, ya Platón, citado por Bedoya (2002), desde la era de la antigua filosofía, proponía despertar, alimentar y desenvolver las facultades del ser humano en un sistema de educación fundamentado en una sociedad regida por la justicia. Por esto, es necesario hacer propuestas innovadoras o renovadoras que transformen la realidad; es preciso revisar los principios fundamentales que dignifican al ser humano y que no favorezcan su explotación. Estas propuestas innovadoras se pueden plantear a través de la construcción de propuestas curriculares mediante la tradición práctica deliberativa y crítica, con una concepción curricular basada en el desarrollo humano y la transformación social que, según Villarini (1996), se complementan en los diferentes niveles curriculares.

Por qué la insistencia en transformar la realidad; por qué desde la educación tratar de que la persona piense, reflexione, analice y proponga, es decir, aprenda con autonomía, en diferentes medios; por qué, como dice Delors et al. (1996), priva la necesidad de aprender en el transcurso de la vida. Hay muchas razones, una de ellas, la cual motiva la discusión, es que, a pesar de que los avances científicos y tecnológicos han impactado la sociedad en todas sus dimensiones y el conocimiento cada vez es más amplio y accesible, las condiciones de vida de las personas cada día se desmejoran. Por ello, los problemas de la realidad deben ser abordados por los sistemas educativos para cambiar y transformar las sociedades.

No se trata de una panacea, pero sí de que, desde la planificación curricular, se propongan reformas, previo estudio de los problemas desde las fuentes curriculares y no prescribir desde los expertos. Se trata de contrastar la educación tradicional con la basada en competencias, desde un diseño con fundamento en la deliberación y la visión crítica de sus artífices, con un enfoque de educación centrado en la persona y con perspectiva social.

El estudio de la realidad, para proponer su transformación a partir de la construcción de proyectos curriculares, es también respaldado por ideas de Moreno (1999), Bolívar (1999), Schwab, citado por Moreno (1999) y Kemmis (1998).

En el diseño del currículo, a partir del insumo recibido de la tradición hermenéutica en investigación educativa, prevalece el análisis cualitativo de la realidad. Lo anterior permite abordar el conocimiento desde las expectativas, creencias y experiencias previas de los participantes y las participantes, quienes juegan un papel importante en el desarrollo curricular. Este pensamiento se 
relaciona con lo dicho por Kemmis (1998), quien manifiesta que la práctica supone un mundo en el que el profesorado debe participar activamente en la toma de decisiones educativas.

De acuerdo con Schawb, citado por Moreno (1999), el campo del currículo se abrió al ámbito de la práctica y a su lenguaje propio, y al reto de comprender la naturaleza. Característica que permite una nueva construcción de los procesos, su configuración y su desarrollo. Ese aporte se denota en la dimensión social del aprendizaje, en los procesos interpersonales que se dan en el aula y en los patrones de valoración de los contenidos. Por ello, la importancia de deliberar sobre la realidad educativa desde el punto de vista de todos sus actores.

Esos modelos de diseño curricular se orientan a la innovación, por medio de la intervención docente, en el papel de la investigación activa, como lo propone Stenhouse, citado por Kemmis (1998). Se considera, en este modelo, la interdisciplinariedad; el desarrollo de las capacidades y habilidades de los alumnos; el desarrollo de actividades democráticas, a modo de ejes del aprendizaje; la orientación cognitiva de aprender a aprender; aprender a pensar; el protagonismo de la persona docente y de la persona que aprende, cada uno con un papel participativo. Prevalecen las experiencias de aprendizaje continuo, por medio de las vivencias y su reconstrucción, mediante procesos de acción-reflexión en los conocimientos integrados.

El diseño curricular desde la perspectiva práctico-deliberativo involucra la opinión, las ideas, los aportes del profesorado; sin embargo, aún falta un poco más de participación de todos los artífices y las artífices del proceso de enseñanza y de aprendizaje. Esa mayor participación en la construcción del currículo y su diseño, desde la integración del saber, el saber hacer, el ser y el convivir para dar respuesta al cierre de la brecha entre educación, trabajo y la vida, encuentra fundamento en la visión crítica del currículo; visión crítica que Bolívar (1999) plantean como la posibilidad de hilar los factores económicos, históricos, culturales y políticos de la educación, basados en las ideas neomarxistas y el apoyo en la Escuela de Frankfort.

El modelo de diseño, desde la visión crítica, complementa la práctica deliberativa, al demostrar que la experiencia y el conocimiento escolares están siempre condicionados a la experiencia. Se deben practicar nuevas formas de leer las relaciones entre la educación, la escuela y la sociedad, en el campo de la política, los valores ideológicos, históricos y los criterios de compromiso ético de liberación y emancipación.

El currículo como construcción social se sitúa, según Moreno (1999), en un espacio social amplio, no solo limitado a las prácticas docentes, sino que profundiza en sus condicionamientos culturales, sociales y políticas. Esos condicionantes se perfilan desde los parámetros del desarrollo humano, con una identidad personal y comunitaria; un currículum común, cultura y valores iguales para todos; el reconocimiento de las diferencias y de la diversidad multicultural, las necesidades materiales y económicas, culturales, la individualidad crítica y la autonomía para el fortalecimiento de valores comunitarios, y la consecución de saberes individuales y la relación con los demás.

Paulo Freire también fundamenta esta perspectiva crítica del diseño curricular -en oposición a la educación bancaria- con el pensamiento crítico sobre los problemas, por medio de una comunidad dialógica entre persona docente y persona estudiante; orientada a problematizar la realidad vivida y susceptible de transformación (Freire, 2006). Esa idea la comparten otros autores sobre la educación democrática y la acción transformadora de la realidad. Entre ellos se pueden citar algunos como Fals (1989) y Montero (2006) quien cita a Grossi, Freire, Cohen, Le Boterf, De Witt, Gianotten, quienes consideran que su aporte a las ciencias sociales se desarrolla en torno a irradiar la acción y la reflexión de la realidad. De esa manera, se reconoce a las personas como sujetos de conocimiento en un contexto teórico-contexto real. 
En oposición con los modelos de diseño, a partir de la deliberación práctica y crítica, las de corte técnico y experto presentan una situación excluyente de la participación de la persona docente, de la persona estudiante y de las condiciones contextuales; de manera que el currículum que prevalece es el prescrito, el cual, por medio de los mecanismos de control y seguimiento en el aula, debe cumplirse al pie de la letra. Esa prescripción motiva a la transmisión de conocimiento y a considerar el proceso de enseñanza y de aprendizaje como un espacio para aprender contenidos, concepción del currículo a base de disciplinas y de destrezas, sin ninguna criticidad de aplicación o adaptación a otras situaciones.

Para el logro de un proyecto curricular con posibilidades de desarrollo de la persona en su libertad, en su accionar y su reflexión para resolver los problemas propios, con las creencias y aportes de la cultura y de la diversidad, requiere un punto de vista de la educación y del currículo más allá de la transmisión de conocimientos, habilidades y destrezas para el desempeño de una tarea en un futuro puesto de trabajo. Kemmis (1998) representa esas ideas desde la teoría del currículo como: un lenguaje y discurso humanista y dialéctico; las relaciones sociales y la organización participativas, democráticas y comunitarias, y la acción y práctica emancipadoras.

Por lo anterior, al plantear proyectos educativos en el enfoque por competencias, que se perfilan como innovadoras en la formación de personas, es importante considerar:

- El diálogo y el consenso de quienes promueven el proceso educativo (profesorado, estudiantado, administración educativa, instituciones comunales, personas contratantes, comunidad, familia), en sus necesidades y exigencias.

- Investigación acción-reflexión, estudiar e intervenir la realidad para transformarla.

- Identificación de problemas individuales, familiares, comunales y la búsqueda de sus soluciones.

- Deliberación profesional (profesionalización de la persona docente para que participe con liderazgo y conocimiento en los procesos de diálogo para establecer proyectos educativos con aporte de los diferentes niveles macro, meso y micro).

- Interacción escuela-comunidad en el estudio de las necesidades individuales, formativas y de intervención social.

- Liderazgo administrativo.

- Liderazgo de la persona docente.

- Visión de la educación con miras a una sociedad regida por la justicia, deliberante y crítica para defender sus fines más esenciales, el desarrollo de los países que pretenden progresar hacia un futuro mejor de sus pueblos y rechazar las prescripciones del interés económico mundial.

- Equilibrio entre los aspectos políticos, económicos y sociales para dar respuesta a los problemas humanos.

- La utilidad del conocimiento y su aplicación a las demandas de propagación de valores por medio de la educación y la necesidad de fomentar un espíritu comunitario.

- Respuesta en términos de profesionalización a los avances científicos y tecnológicos, la apertura de mercados, la multiculturalidad, la visión planetaria, sin dejar de lado la comprensión del significado del individuo consigo mismo, con los otros y la globalidad.

- Participación desde adentro, de los creadores y las creadores del proyecto educativo (fuentes curriculares).

Estos son factores de un currículum basado en el desarrollo humano, cuya concepción humanista, según Villarini (1996), convierte la atención en la experiencia y necesidades de la 
persona estudiante y a sus perspectivas biopsicosocial y cuyo fin principal es el desarrollo integral en las dimensiones que Bruner, Maslow, Piaget y Vigostsky aportan en los ámbitos intelectual, emocional, social y psicomotor. Por tanto, la estructura curricular es abierta y flexible. Esta concepción curricular se complementa con la realidad social, cultural y política del estudiantado. Constituye un currículo basado en la transformación social, que tiene como objetivo desarrollar, en la persona, una comprensión crítica y un compromiso de transformación.

\section{Reflexión final}

Los proyectos educativos basados en el desarrollo de competencias deben centrarse en la persona que aprende y focalizar el proceso de enseñanza y de aprendizaje en el fortalecimiento de sus capacidades y habilidades, en un contexto ilimitado en tiempo y espacio.

Además, tomar en cuenta que el contexto en el que está inmersa la persona estudiante es incierto, pues en el que prevalece la visión capitalista del conocimiento y nuevas relaciones socioculturales; fundamentado en el uso de nuevas tecnologías de la comunicación y la información, donde la flexibilidad y adaptabilidad son capacidades inmediatas.

En ese nuevo contexto económico-productivo, de relaciones sociales, religiosas, políticas, laborales o profesionales, es prudente repensar la estructura de los proyectos educativos, de manera que se visualice la formación de personas con capacidad de analizar, de recolectar, de sintetizar información, de indagar los problemas de la realidad inmediata, nacional e internacional, de proponer soluciones y de transformarla.

Ese conocimiento de la persona, guiado desde la educación, obligatoriamente, debe fundamentarse en los cuatro pilares de la educación, para que cada persona logre en el transcurso de la vida: aprender a conocer para una vida plena, a partir del desarrollo de las capacidades de interacción con los demás; aprender a hacer, a través de un conjunto de competencias específicas de cada persona, en los ámbitos de la formación técnica y profesional, los comportamientos y las aptitudes para trabajar en forma individual y en equipo; aprender a vivir con los demás en función del respeto de la diversidad y de la interdependencia, la solidaridad y la convivencia en paz; aprender a ser considerando el equilibrio entre el cuerpo, la mente y el espíritu.

En educación, las preguntas: qué, cómo y para qué remiten a la deliberación profunda del sentido de un proceso formativo frente a una cultura del aprendizaje del siglo XXI. Al respecto, Pozo et al. (2006) consideran que un nuevo reto para las creencias más profundas sobre el aprendizaje, caracteriza esa nueva cultura con tres rasgos fundamentales: la sociedad de la información, del conocimiento múltiple e incierto y del aprendizaje continuo.

La realidad cambiante, exigente, incierta, compleja, realista y global obliga a la reflexión y autorreflexión de los sistemas educativos, en términos de qué se enseña, cómo son los procesos de enseñanza y para qué se está enseñando: ¿será lo que un programa de curso dicta?, y en ese proceso, ¿qué, cómo y para qué están aprendiendo el estudiantado?

Con lo anterior, no se pretende decir que la educación es la responsable de todos los males de la humanidad, ni que la enseñanza por competencias debe ser planificada desde un enfoque u otro. No es una panacea para los males de la educación, pero sí resulta importante reflexionar sobre el hecho de que en el ámbito educativo se perfilen y defiendan proyectos para mejorar la calidad de vida del ser humano. Para ello, las personas diseñadoras, quienes ejercen la administración educativa y ejecutan el currículo deben tener una visión deliberante y crítica para defender los fines más esenciales de la educación: el desarrollo de los países que pretenden evolucionar hacia 
un futuro mejor de sus pueblos y no aceptar las prescripciones del interés económico mundial. Esto podría ser novedoso ante la actual realidad, estudiarla y, a partir de la solución de los problemas, transformarla.

\section{Referencias bibliográficas}

Alpízar, J. L. (2008). ¿Profesionales competitivos o competentes? II. Tipología de competencias. Revista Pedagogía Universitaria, 13(4), 1-14. Recuperado de http://www.buenastareas.com/ ensayos/Profesionales-Competentes/1859945.html

Arias, A. y Charpentier, S. (2004). Diccionario de competencias laborales: la experiencia en el sector bancario público costarricense (Tesis de grado). Universidad de Costa Rica, San José, Costa Rica.

Bedoya, J. I. (2002). Epistemología y pedagogía: ensayo histórico crítico sobre el objeto y método pedagógicos ( $5^{\mathrm{a}}$ ed.). Colombia: Ecoe.

Bolívar, A. (1999). Modelos de diseño curricular de corte crítico y postmoderno: descripción y balance. En J. M. Escudero, [Coord]. Diseño, desarrollo e innovación del currículum (pp. 145164). Madrid, España: Editorial Síntesis, S. A.

Catalano, A. M., Avolio, S. y Sladogna, M. (2004). Diseño curricular basado en normas de competencia laboral. Conceptos y orientaciones metodológicas. Buenos Aires: Banco Interamericano de Desarrollo.

Coll, C. (1991). Psicología y currículum. Una aproximación psicopedagógica a la elaboración del currículum escolar. Barcelona, España: Padiós.

Delors, J., Mufti, I., Amagi, I., Carneiro, R., Chung, F., Geremek, B., ... y Nanzhao, Z. (1996). La educación encierra un tesoro. Informe a la UNESCO de la Comisión Internacional sobre la Educación para el siglo XXI (Compendio). Francia: Santillana Ediciones UNESCO.

Díaz, F. (2005). Desarrollo del currículo e innovación: modelos e investigación en los noventa. Perfiles Educativos, 27(107), 57-84.

Fals, O. (1989). El problema de cómo investigar la realidad para transformarla por la praxis $\left(7^{\mathrm{a}}\right.$ ed.). Bogotá, Colombia: Tercer mundo editores.

Freire, P. (2006). Pedagogía de la autonomía. Saberes necesarios para la práctica educativa (Traducción de G. Palacios) (3 ${ }^{\text {a }}$. Reimp.). Buenos Aries, Argentina: Siglo XXI Editores Argentina S. A.

Gimeno, J. (2009). Introducción. En J. Gimeno (Comp.). Educar por competencias, ¿qué hay de nиеvo? (pp. 9-13). Madrid, España: Ediciones Morata, S. L. 
González, V. (2005). Tecnología digital: Reflexiones pedagógicas y socioculturales. Revista Electrónica Actualidades Investigativas en Educación, 5(1), 1-24, Recuperado el 28 de enero de 2010. Recuperado de http://revista.inie.ucr.ac.cr/articulos/1-2005/articulos/digital.pdf

Kemmis, S. (1998). El curriculum: más allá de la teoría de la reproducción (Trad. P. Manzano). Madrid, España: Ediciones Morata, S. L.

Levy-Levoyer, C. (1997). Gestión de las competencias, cómo analizarlas, cómo evaluarlas, cómo desarrollarlas. Barcelona, España: Ediciones Gestión 2000, S. A.

Mertens ( 2000). La gestión por competencia laboral en la empresa y la formación profesional. Madrid, España: Organización de Estados Iberoamericanos para la Educación, la Ciencia y la Cultura (OEI), Recuperado de http://www.oei.es/oeivirt/fp/iberfop01.htm

Montero, M. (2006). Hacer para transformar: El método en la psicología comunitaria. Buenos Aires, Argentina: Ediciones Paidós.

Morales, G. (2009). El claroscuro de la formación por competencias. Xipe Totek, 18(71), 193-215.

Moreno, J. M. (1999). Modelos de corte deliberativo y práctico:descripción y balance. En J. M. Escudero, [Coord]. Diseño, desarrollo e innovación del currículum (pp. 123-144). Madrid, España: Editorial Síntesis, S. A.

Pérez, Á. I., (2009). ¿Competencias o pensamiento práctico?: la construcción de los significados de representación y de acción. En J. Gimeno (Comp). Educar por competencias, ¿qué hay de nuevo? (pp. 59-102). Madrid, España: Ediciones Morata, S. L.

Pozo, J. I., Scheuer, N., Pérez, M., Mateos, M., Martín, E. y de la Cruz, M. (2006). Nuevas formas de pensar la enseñanza y el aprendizaje. Las concepciones de profesores y alumnos. Barcelona. España: Editorial Graó.

Proyecto Tuning América Latina (2005). “Tuning” Afinar las estructuras educativas de Europa. (Proyecto piloto apoyado por la Comisión Europea en el marco del programa Sócrates). Recuperado de http://www.tuning.unideusto.org/tuningal/

Riesco, M. (2008). El enfoque por competencias en el EEES y sus implicaciones en la enseñanza y el aprendizaje. Tendencias Pedagógicas, 13, 79-105. Recuperado de http://dialnet.unirioja.es/ servlet/articulo?codigo $=2575310$

Tobón, S. (2005). Formación basada en competencias (2a. ed.). Bogotá, Colombia: Ecoe Ediciones.

Tünnermann, C. (2008). Introducción. En C. Tünnermann (Ed.). La educación superior en América Latina y el Caribe: diez años después de la Conferencia Mundial de 1998 (pp. 11-44). Cali, Colombia: Iesalc-Unesco. Recuperado de http://www.iesalc.unesco.org.ve 
Vargas, J. G. (s. f.). Las reglas cambiantes de la competitividad global en el nuevo milenio. Las competencias en el nuevo paradigma de la globalización. OEI. Revista Iberoamericana de Educación, 1-20. Recuperado de http://www.rieoei.org/deloslectores/186Vargas.PDF

Villa, A. y Poblete, M. (2004). Practicum y evaluación de competencias. Profesorado. Revista de currículo y formación del profesorado, 8(2), 1-19. Recuperado de http://redalyc.uaemex.mx/ $\underline{\text { src/inicio/ArtPdfRed.jsp?iCve }=56780203}$

Villarini, A. R. (1996). El currículo orientado al desarrollo humano integral. Puerto Rico: Biblioteca del Pensamiento Crítico. 